\title{
Physics-Informed Neural Network with Variable Initial Conditions
}

\author{
Yo Nakamura $^{1}$, Suguru Shiratori ${ }^{1}$, Hideaki Nagano ${ }^{1}$, Kenjiro Shimano ${ }^{1}$ \\ ${ }^{1}$ Mechanical Systems Engineering, Tokyo City University \\ 1-28-1 Tamazutsumi, Setagaya-ku, Tokyo, Japan \\ g2081038@tcu.ac.jp (Y. Nakamura); sshrator@tcu.ac.jp (S. Shiratori)
}

\begin{abstract}
This work addresses an extension of Physics-Informed Neural Network (PINN), which learns solutions of partial differential equations (PDE). In the originally proposed PINN [1], the initial conditions are kept constant. In this study, we extend the PINN so that the initial conditions can be varied by means of low-dimensional identifiers which represent the initial conditions. A validity of the proposed method is confirmed through the PDE for the liquid film flow. The solutions of the PDE predicted by the PINN showed good agreement with those obtained by the finite difference method, and the dependence of the initial conditions are also correctly reproduced. As requirements for the low-dimensional identifier of the initial conditions, it is suggested that continuity and uniqueness are necessary condition and the linearity is sufficient condition.
\end{abstract}

Keywords: 4 - 8 keywords

Machine learning, Numerical simulation, Liquid film flow, Partial differential equations

\section{Introduction}

In the manufacturing process of semiconductors and MEMS devices, a resist film consisting of a resin and a solvent is coated onto a substrate, and it is known that various thickness undulations appear in the final resist films [2, 3]. Although numerical simulations have been used to predict such thickness undulations, it is difficult to optimize the coating conditions from an inverse problem perspective because the time evolution of the governing equations takes a long calculation time. In the general optimization procedure, it is necessary to evaluate the objective function and its gradient with respect to the parameters in coating conditions. Both take much computational cost in the conventional numerical method based on the time evolution calculation.

Physics-Informed Neural Network (PINN), which has been proposed by Raissi et al. [1], can be considered as a way to resolve such a situation. The PINN learns the solutions of a partial differential equation (PDE) for given dataset of the input variables. In the training process of PINN, a loss function is defined as the mean square error of the predicted solutions of the PDE. To evaluate the loss function, the temporal and spatial derivatives of the unknowns are calculated by the automatic differentiation (AD) which is implemented in the neural network (NN) framework. Once a PINN has been trained, the solutions for any time instance can be directly calculated without time integration by forward calculations of the NN. In addition, the gradient of the solution with respect to the input variable can be efficiently calculated by the AD. The authors' research group has studied to apply the PINN to the PDE for the liquid film flow in order to finally achieve the optimization of the coating conditions which can avoid or suppress the thickness undulations. In the problem of the liquid film flow including the surface tension (Laplace pressure) effect, the PDE involves a fourth-order spatial derivative and the fourthorder nonlinearity of unknowns. Even for such a type of PDE, the PINN was found to correctly predict the solutions [4].

In the original PINN, the initial conditions are fixed during training. For the different initial conditions, the PINN has to be retrained. Due to this restriction, the it is difficult to calculate the gradient of the solution with respect to the initial conditions, thus the PINN cannot be applied to the optimization of the initial conditions of the coating process. The PINN is expected to also be applied to the data assimilation, in which the uncertain parameters for the simulation are optimized to minimize the difference between simulation and observation [5]. Regarding the application of the PINN to the data assimilation, the restriction of initial conditions becomes to be serious drawback.

Therefore, the purpose of this study is to extend the original PINN so that the initial conditions be variable. A way to realize this is described in the next section, then the proposed method is validated by comparing the results with those obtained by the finite difference method. 


\section{Approach}

\subsection{Overview}

Figure 1 shows how we make the initial condition be variable in the PINN. We express the initial conditions with low-dimensional identifiers. These identifiers are obtained by certain mapping from the initial conditions. Then, these identifiers are taken as input of the PINN. During training of the PINN, the loss function is evaluated using not the lowdimensional identifier but the original initial condition itself. Although, in principle the discretized initial conditions can be directly put into the PINN, the number discretized points are likely be too huge for the input of the PINN. We consider making the low-dimensional identifiers in order to reduce the input dimension of the PINN.

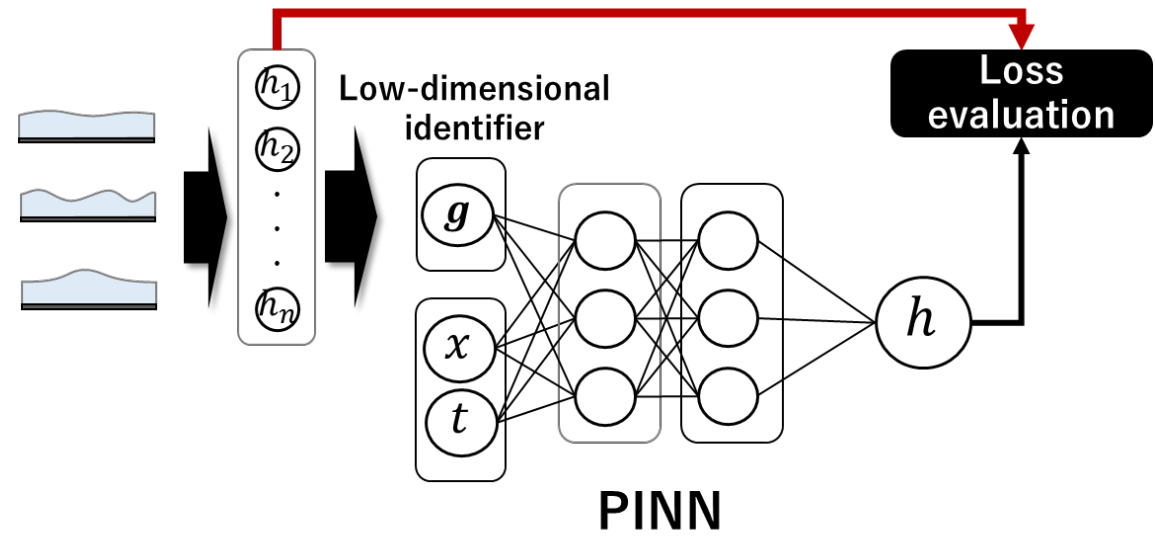

Fig. 1: PINN with a low-dimensional identifier added to the input

\subsection{Low-Dimensional Identifiers}

In this study, we considered four types of low-dimensional identifiers; LIN, CAE, WAV and DIS, as summarized in Table 1. The LIN is the identity mapping, where the initial conditions are expressed as parametric analytic function, and its parameters are directly used as the identifier. This LIN is regarded as essential verification whether the difference in initial conditions can be reflected in the PINN. In the CAE, the given dataset of initial conditions is mapped onto the identifiers by the Convolutional Autoencoder (CAE) [6]. Through the CAE network, the data dimension of the initial condition can be highly reduced. In the CAE, we examine the extensibility of this method to general-purpose input. WAV creates a non-linear low-dimensional identifier and examines the effect of the degree of non-linearity. The WAV is a nonlinear mapping with a wavy function Through this WAV, we investigate the effect of nonlinearity in the accuracy of the PINN. Finally, the DIS is the discontinuous mapping. The function used as DIS is discontinuous but unique (no folding). Through this DIS, we will investigate whether the continuity is necessary for the identifiers. Figure 2 shows the detailed functions used as mapping of the identifiers.

Table 1: Low-dimensional identifiers considered

\begin{tabular}{|c|c|c|}
\hline Identifier & Description & Function \\
\hline LIN & Identity mapping & $f(x)=x$ \\
\hline CAE & Encoded by CAE & $f(x)=\operatorname{CAE}(x)$ \\
\hline WAV & Nonlinear mapping & $f(x)=x+\frac{1}{20} \sin (6 \pi x)$ \\
\hline DIS & Discontinuous mapping & $f(x)=\left\{\begin{array}{cc|}1-x & (x \leq 1 / 8) \text { or }(1 / 4<x \leq 3 / 8) \\
x+1 / 8 & \text { else }\end{array}\right.$ \\
\hline
\end{tabular}



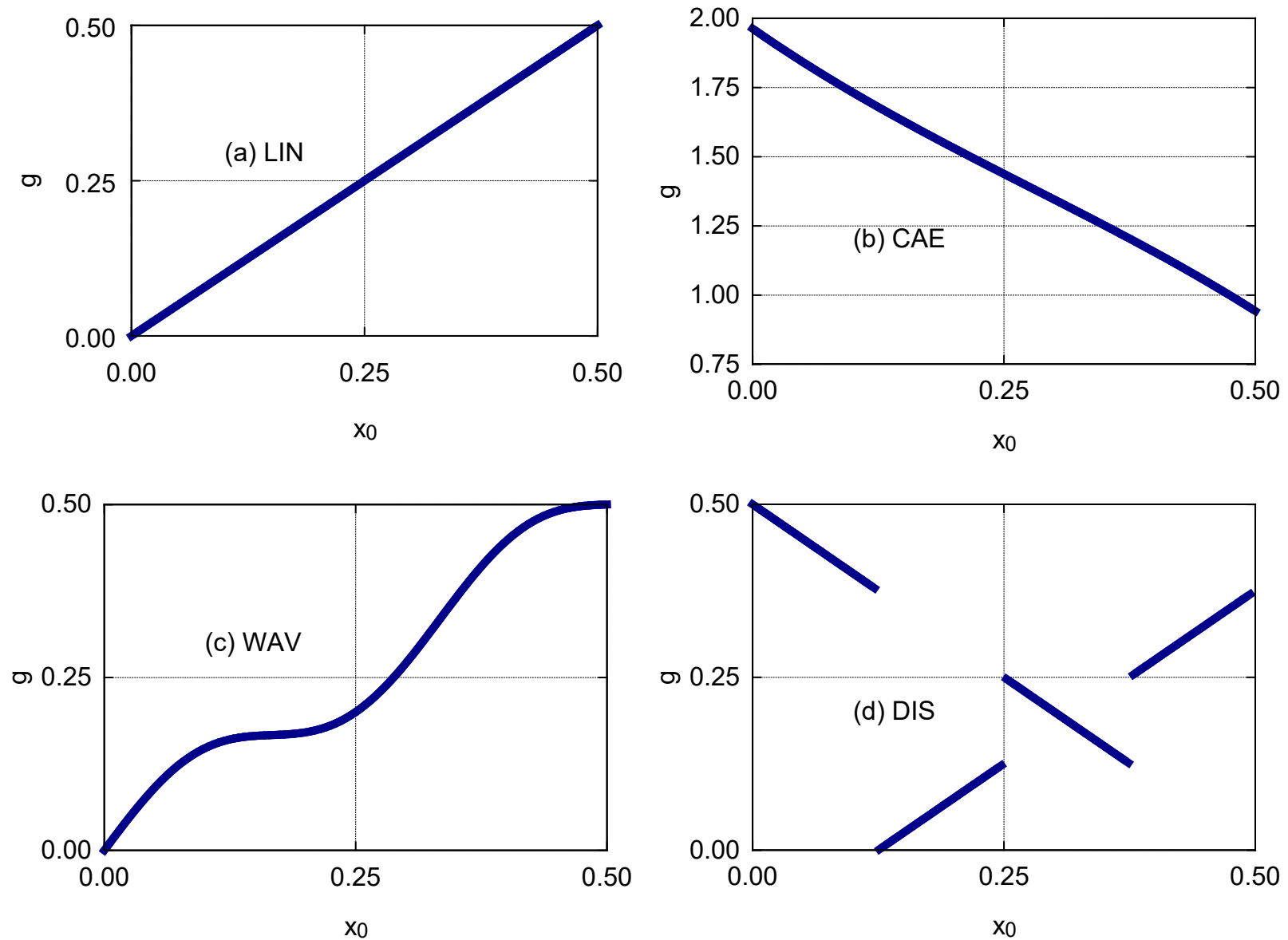

Fig. 2: Profiles of mapping functions for low-dimensional identifiers. (a) linear function: LIN, (b) encoded by CAE, (c) wavy function: $\mathrm{WAV}$, and (d) discontinue function: DIS.

\section{Validation}

\subsection{Problem formulation: Governing equation and boundary conditions}

In order to validate the proposed method, it is applied to a liquid film flow problem. As the governing equations, we consider the following equations, where only the relaxation term due to Laplace pressure is considered.

$$
F: \frac{\partial h}{\partial t}+\frac{\partial}{\partial x}\left(h^{3} \frac{\partial^{3} h}{\partial x^{3}}\right)=0
$$

This partial differential equation describes the time evolution of the film thickness distribution due to surface tension. $h, t$, and $x$ are the film thickness, time, and coordinates, respectively. The equation is nondimensionalized using the scales listed in the Table 2, where $h_{0}$ is the base thickness of the film, $L$ is the representative length, $\mu$ is the viscosity coefficient and $\sigma$ is the surface tension.

Table 2: Scales used for the nondimensionalization.

\begin{tabular}{|c|c|c|c|}
\hline Lateral length & Thickness & Time & Pressure \\
\hline$L$ & $h_{0}$ & $3 \mu L^{4} /\left(\sigma h_{0}^{3}\right)$ & $h_{0} \sigma / L^{2}$ \\
\hline
\end{tabular}


The following boundary conditions were applied so that the gradient of the thickness and the curvature becomes zero at the end of the solution domain.

$$
B: \frac{\partial h}{\partial x}=\frac{\partial^{3} h}{\partial x^{3}}=0 \quad \text { at } \quad x=x_{\min }, \quad x_{\max }
$$

\subsection{Initial Conditions}

The initial conditions are expressed in the following functional form.

$$
S: h(x, t=0)=\left(h_{1}-h_{0}\right)\left(1-\left(x-\frac{x_{0}}{\lambda}\right)^{2}\right) \exp \left(\frac{\left(x-x_{0}\right)^{2}}{\left(2 \lambda^{2}\right)}\right)+h_{0}
$$

Using Eq. (3) and the parameters listed in the Table 3, the dataset of the initial conditions are generated. The number of discrete points in the initial condition is selected as $N_{x}=256$.

Table 3: Dataset of initial condition parameters

\begin{tabular}{|c|c|c|c|c|}
\hline Parameter & $x_{0}$ & $\lambda$ & $h_{0}$ & $h_{1}$ \\
\hline Value range & $0.0 \sim 0.5$ & 0.7 & 1 & 3 \\
\hline
\end{tabular}

\subsection{Loss Function of PINN}

The loss function of PINN is defined as follows.

$$
\begin{aligned}
& J_{P I N N}=J_{F}+J_{O}+J_{P}+J_{L_{2}} \\
& J_{F}=\sum_{i}^{N_{f}}\left[F\left(h_{P I N N}\left(x_{i}, t_{i}, \boldsymbol{g}_{i}\right)\right]^{2}\right. \\
& J_{O}=\sum_{i}^{N_{\text {ini }}}\left[h_{\text {PINN }}\left(x_{i}, t_{i}, \boldsymbol{g}_{i}\right)-h_{\text {ini }}\left(x_{i}\right)\right]^{2} \\
& J_{B}=\sum_{i}^{N_{B}}\left[\left.\frac{\partial h}{\partial x}\right|_{P I N N}\left(x_{\text {min,max }}, t_{i}, \boldsymbol{g}_{i}\right)\right]^{2}+\sum_{i}^{N_{B}}\left[\left.\frac{\partial^{3} h}{\partial x^{3}}\right|_{P I N N}\left(x_{\text {min }, \text { max }}, t_{i}, \boldsymbol{g}_{i}\right)\right]^{2} \\
& J_{P}=\sum_{i}^{N_{f}} \max \left(0, \exp \left(-h_{P I N N}\left(x_{i}, t_{i}, g_{i}\right)\right)-1\right)
\end{aligned}
$$

$J_{F}$ is the mean squared error of the governing equation, $J_{O}$ and $J_{B}$ are the initial and boundary conditions, respectively, $J_{P}$ is the penalty function for the film thickness $h$ to impose a positivity constraint, and $J_{L_{2}}$ is the L2 normalization term. $\boldsymbol{g}$ is low-dimensional identifier for the initial conditions. In the present study, the network is composed by 8 hidden dense layers with 20 neurons per layer. The training dataset was generated by randomly sampled $N_{f}=3000$ points from a set of discrete points equally spaced on a linear scale in the $x$ direction and on a logarithmic scale in the $t$ direction.

\subsection{Finite Differences}

In order to validate the proposed method, the results obtained by the PINN was compared with those obtained by the finite difference method (FDM). In the FDM, the governing equation Eq. (1) is discretized by the finite differences on a equidistant grid of $N_{x}$ points with an interval $\Delta x$. The positivity-preserving scheme is applied to $h^{3}$ of the Laplace 
pressure term in Eq. (1), because the standard finite difference schemes do not necessarily preserve a positivity of $h$, and the occurrence of nonpositive solution introduces artificial instability [7]. Due to the fourth-order spatial derivative of $h$ in Eq. (1), a time step restriction for the numerical stability requires $\Delta t<O\left(\Delta x^{4}\right)$, which is a quite severe condition in practice. In order to cope with this restriction, the fully implicit time integration of Newton-Kantrovich method is applied with CrankNicholson, according to Diez and Kondic [8].

\section{Results}

Fig. 3 shows the contours of the film thickness in logarithmic scale $\log h$ in the plane of horizontal axis is time $t$ and the vertical axis is coordinate $x$. Fig.3(a) shows the result of FDM, and Fig.3(b) of PINN for the case of the LIN as the lowdimensional identifier. From Fig.3, it can be confirmed that the relaxed shape of the initial condition can be reproduced even if the low-dimensional identifier is used for input.

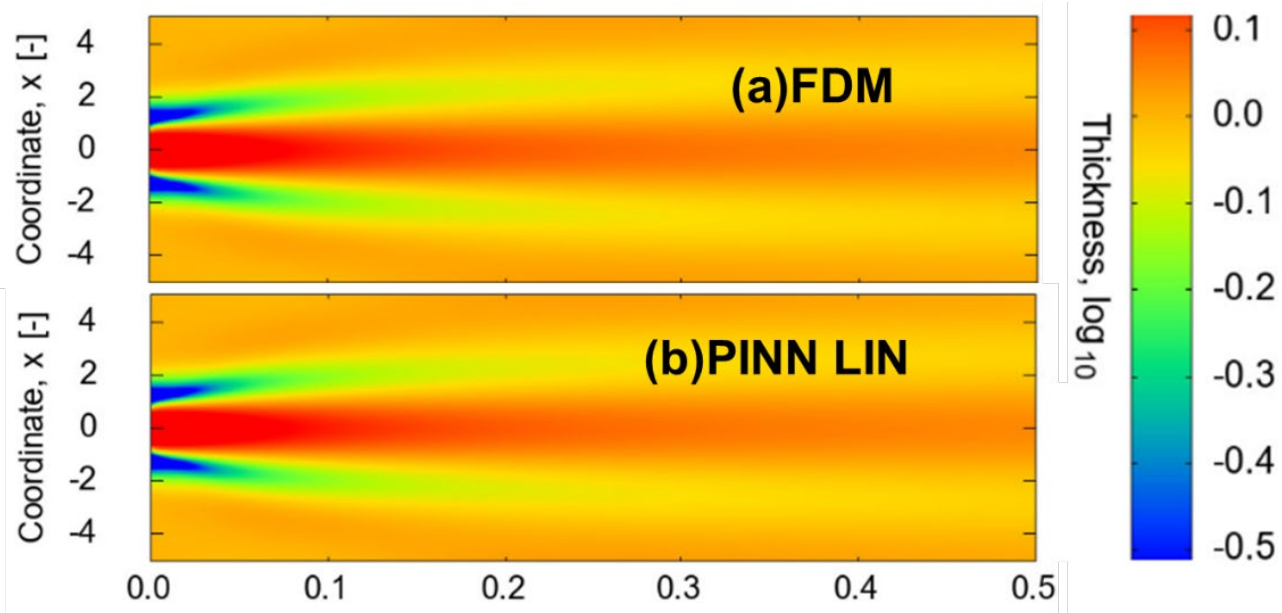

Fig. 3: Prediction of film thickness $h(t, x)$ in space-time $(t, x)$.

(a) FDM (b) PINN with low-dimensional identifier by LIN

For a more detailed comparison, snapshots of the film thickness distributions for the initial state $(t=0)$ and after time $(t=0.5)$ are shown in Fig.4 and Fig.5. Table 4 shows the RMS average values at the initial state $(t=0)$ and at the sufficiently later time instance $(t=0.5)$. From Fig.4, it was found that the difference of the initial conditions is successfully reproduced for the cases of LIN, CAE, and WAV. However, DIS did not reflect the difference in the initial conditions. For the cases of LIN, CAE and WAV, the error between the PINN and the FDM are found at the location where the curvature of the thickness is large. The RMS average in the DIS is one order of magnitude larger than those in other three cases.

Table 4: The RMS average values in the initial state $(t=0)$ and after the passage of time $(t=0.5)$

\begin{tabular}{|c|c|c|c|c|}
\hline Identifier & $\begin{array}{c}\text { Initial state } \\
x_{0}=0\end{array}$ & $\begin{array}{c}\text { Initial state } \\
x_{0}=0.3\end{array}$ & $\begin{array}{c}\text { End state } \\
x_{0}=0\end{array}$ & $\begin{array}{c}\text { End state } \\
x_{0}=0.3\end{array}$ \\
\hline LIN & $4.4 \times 10^{-2}$ & $4.4 \times 10^{-2}$ & $8.8 \times 10^{-3}$ & $9.6 \times 10^{-3}$ \\
\hline CAE & $4.1 \times 10^{-2}$ & $4.2 \times 10^{-2}$ & $9.8 \times 10^{-3}$ & $9.7 \times 10^{-3}$ \\
\hline WAV & $4.8 \times 10^{-2}$ & $4.8 \times 10^{-2}$ & $1.4 \times 10^{-2}$ & $1.7 \times 10^{-2}$ \\
\hline DIS & $1.4 \times 10^{-1}$ & $2.0 \times 10^{-1}$ & $1.2 \times 10^{-2}$ & $2.4 \times 10^{-2}$ \\
\hline
\end{tabular}



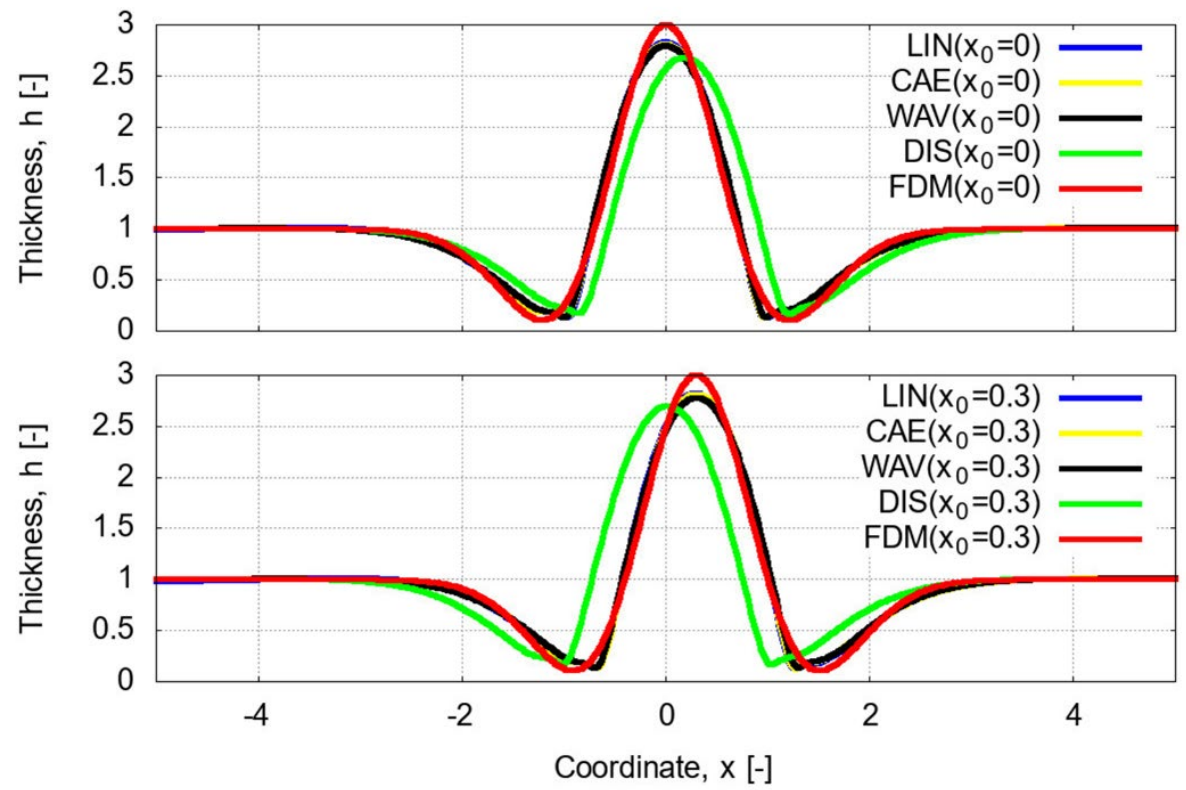

Fig. 4: Snapshots of the thickness distribution at the initial state $(t=0)$ when low-dimensional identifiers of LIN, CAE, WAV, DIS and FDM.
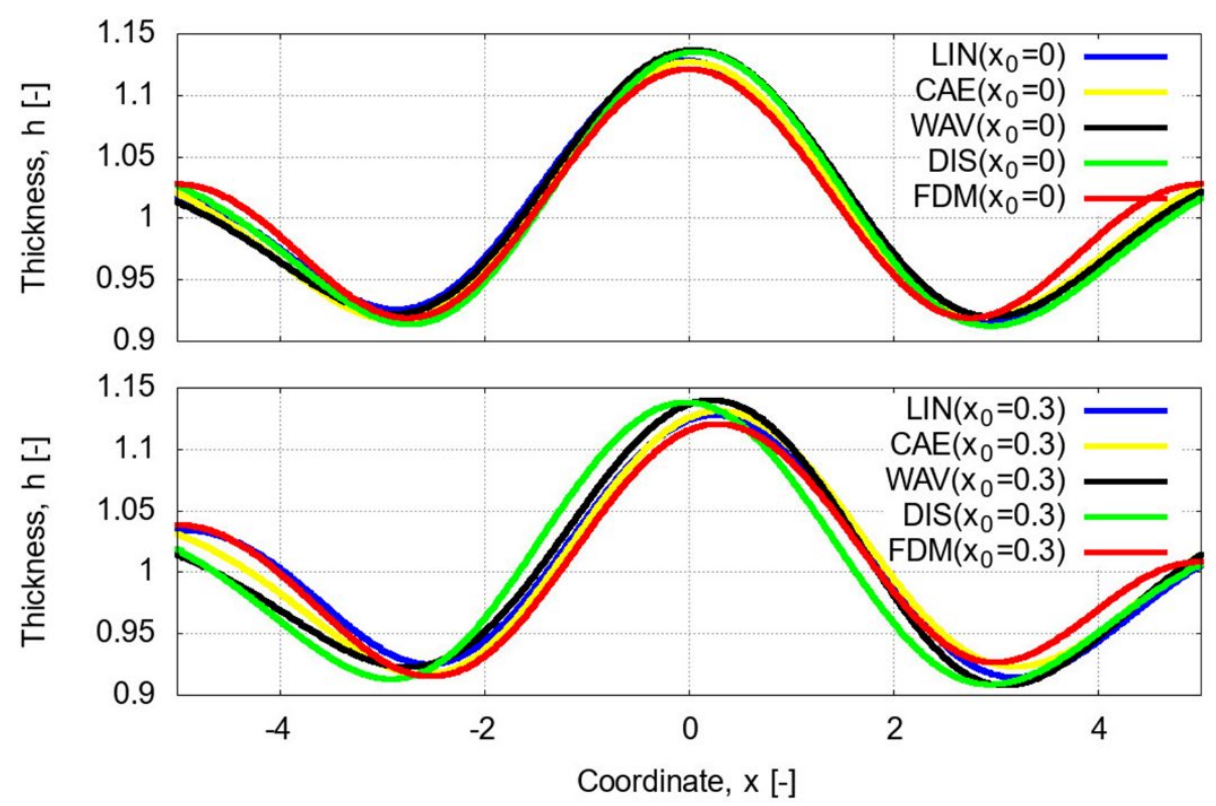

Fig. 5: Snapshots of the thickness distribution at the end state $(t=0.5)$ when low-dimensional identifiers of LIN, CAE, WAV, DIS and FDM. 


\section{Discussion}

From the result shown in Fig.4, Fig.5, and Table 4, it was found that the variation of the initial condition is successfully successfully reproduced by the PINN by the method proposed in the present work; low-dimensional identifiers. However, the accuracy was different depending on the mapping function used. Therefore, we consider what the conditions are required required for low-dimensional identifiers. First, if there are duplicate values in the low-dimensional identifier, they will have have the same low-dimensional identifier value for different initial conditions, and it will not correspond to the difference in the initial conditions, so there should be no duplication (uniqueness of the identifier). From Fig.2, if the discontinuity is involved in the mapping function, the difference in the initial conditions cannot be correctly reproduced (case DIS). Therefore, continuity can be regarded as necessary condition for the low-dimensional identifier. Among the three cases where the initial conditions can be reflected, the difference was the degree of non-linearity. The case LIN is linear, the CAE is slightly nonlinear, and the WAV is highly nonlinear. The prediction error in the case WAV is significant. Although the RMS error are almost the same in cases LIN and CAE, the CAE is more applicability for the general initial conditions.

\section{Conclusions}

In the present study, the PINN is extended so that the initial condition can be varied by the low-dimensional identifiers. Through the validation of the four-types of the identifier, we concluded that continuity and uniqueness are necessary condition and the linearity is sufficient condition for the low-dimensional identifier of the initial conditions. Even if these requirements for the identifier are satisfied, the accuracy of the PINN is depending on the degree of the nonlinearity of the mapping functions.

By use of the proposed method, it can be realized that the gradient of the solutions with respect to the initial conditions. Consequently, the optimization of the process conditions in the film-coating is expected to be realized.

\section{References}

[1] M. Raissi, P. Perdikaris and G.E. Karniadakis "Physics-informed neural networks: A deep learning framework for solving forward and inverse problems involving nonlinear partial differential equations," J. Comp. Phys. vol. 378 686707, 2019.

[2] S. Shiratori, D. Kato, K. Sugasawa, H. Nagano and K. Shimano "Spatio-temporal thickness variation and transient Marangoni number in striations during spin coating," Int. J. Heat Mass Trans., vol. 154 119678, 2020.

[3] S. Shiratori and T. Kubokawa "Double-peaked edge-bead in drying film of solvent-resin mixtures," Phys. Fluids vol. 27, 102105, 2015.

[4] Y. Nakamura, S. Shiratori, R. Takagi, M. Sutoh, H. Nagano and K. Shimano "Physics-informed neural network applied to $4^{\text {th }}$ order partial differential equation for liquid film flows," Comp. Fluids. (to appear), 2021.

[5] W. Lahoz, B. Khattatov and R. Ménard "Data Assimilation: making sense of observations", Springer, 2010.

[6] W. Liu, Z. Wang, X. Liu, N. Zeng, Y. Liu and F.E. Alssadi "A survey of deep neural network architectures and their applications", Neurocomputing., vol. 234 11-26, 2017.

[7] J.A. Diez, L. Kondic and A. Bertozzi "Global models for moving contact lines," Phys. Rev. E Vol. 63, $011208,2000$.

[8] J.A. Diez and L. Kondic "Computing three-dimensional thin film flows including contact lines," J. Comp. Phys. vol. 183, 274-306, 2002. 